\title{
Leg circles no Cadillac: efeito de diferentes posições de mola na ativação de estabilizadores do tronco
}

\author{
Leg circles on Cadillac: effect of different spring positions on the activation \\ of stabilizers in the trunk
}

DOI: $\underline{\text { http://dx.doi.org/10.36453/2318-5104.2019.v17.n2.p153 }}$

\author{
Catiane Souza ${ }^{1}$, Edgar Santiago Wagner Neto $^{1}$, Fabiane de Oliveira Brauner ${ }^{2}$, \\ Debora Cantergi ${ }^{3}$, Willian Dhein ${ }^{1}$, Jefferson Fagundes Loss ${ }^{1}$ \\ ${ }^{1}$ Universidade Federal do Rio Grande do Sul (UFRGS) \\ 2Pontifícia Universidade Católica do Rio Grande do Sul (PUCRS) \\ ${ }^{3}$ Faculdade de Educação e Tecnologia Iracema (FAETI)
}

\begin{abstract}
RESUMO
Introdução: A correta ativação da musculatura estabilizadora do tronco é essencial em diversas situações, inclusive prevenção e tratamento de lombalgias. A ativação adequada desses músculos é um princípio do Método Pilates, porém nem todos os efeitos das variações dos exercícios estão descritos da literatura. Objetivo: comparar a atividade elétrica de músculos do power house (reto abdominal, oblíquo externo, oblíquo interno/transverso abdominal e multífido) durante a execução do exercício Leg Circles no aparelho Cadillac com mola alta e com mola baixa. Métodos: Foram selecionadas 10 instrutoras de Pilates, 30 anos ( \pm 5$), 58 \mathrm{Kg}( \pm 7)$, estatura $163 \mathrm{~cm}( \pm 7)$ que foram submetidas a testes de contrações isométricas voluntárias máximas, e logo após, à realização do Leg Circles no Cadillac com a mola alta e baixa. Foi coletada a atividade elétrica dos músculos reto abdominal, oblíquo interno/transverso abdominal, oblíquo externo e multífido. Resultados: A ativação do oblíquo interno foi maior na mola alta $(p=0,002)$, assim como a ativação do multífido $(p=0,042)$. Já o oblíquo externo foi mais ativado na mola baixa $(p=0,001)$. $O$ reto abdominal não variou sua ativação $(p=0,375)$. Conclusão: A mola alta pode ser acatada como a posição mais adequada para ativar a musculatura profunda do tronco, visto que nesta situação houve maior ativação do multífido e do oblíquo interno/transverso abdominal, somados à menor ativação do oblíquo externo e a baixa ativação no reto abdominal encontrados na situação com mola alta.
\end{abstract}

PALAVRAS-CHAVE: Biomecânica; Pilates; Eletromiografia; Core.

\section{ABSTRACT}

Background: The correct activation of the trunk stabilizing muscles is essential in several situations, including prevention and treatment of low back pain. Proper activation of these muscles is a principle of the Pilates Method, but not all the effects of exercise variations are described in the literature. Objective: compare the electric activity of power house muscles (rectus abdominis, external oblique, internal oblique/transverse abdominal and multifidus) during the execution of the Leg Circles exercise with high spring and low spring in Cadillac apparatus. Methods: Ten Pilates instructors were selected, with 30 years $( \pm 5), 58 \mathrm{~kg}( \pm 7), 163 \mathrm{~cm}( \pm 7)$ who underwent maximum voluntary isometric contraction tests before the execution of the Leg Circles on the Cadillac, with high and low spring. The electrical activity of the rectus abdominis, internal oblique/transverse abdominal, external oblique and multifidus muscles was collected. Results: The internal oblique $(p=0,002)$ and the multifidus $(p=0,042)$ activations were greater on the high spring. However, the external oblique showed a higher activation on the lower spring $(p=0.001)$. The abdominal rectus did not change its activation $=0.375)$. Conclusion: The high spring can be considered as the most suitable position to activate the deep musculature of the trunk, since the greater activation of the multifidus and of the internal oblique/transverse abdominal, together with the lower activation of the external oblique and the low activation in the rectus abdominus found in the high spring situation.

KEYWORDS: Biomechanics; Pilates; Electromyography; Core. 


\section{INTRODUÇÃO}

Um controle adequado na ativação dos estabilizadores do tronco, em especial dos músculos transverso do abdome e multífidos, é essencial para a integridade da coluna lombar (FRANÇA et al., 2008; MARQUES et al., 2013). Por exemplo, pacientes com dor lombar apresentam um atraso no início da contração do transverso abdominal (HODGES et al, 1996) e inibição dos multífidos (HIDES et al, 1994). A ativação desses músculos vem sendo usada há décadas a partir da estabilização segmentada (RICHARDSON; JULL, 1995), uma vez que a maior estabilização da região previne e alivia a dor (FRANÇA et al., 2008). Sendo assim, busca-se a ativação dos músculos profundos de forma isolada, pois essa estratégia é apontada como mais eficiente do que sua ativação em conjunto com músculos superficiais, essa ativação, considerada correta, dos estabilizadores profundos do tronco, possibilita, inclusive, diminuir as recidivas de quadros álgicos (RICHARDSON et al, 2002).

Sabe-se que exercícios que incentivam de forma funcional a musculatura do tronco vem sendo preconizados para atingir os músculos mais profundos da região (McGIII, 2001). Considerando que a estabilidade dos segmentos proximais é necessária para a mobilidade dos segmentos distais (SHERRY; BEST; HEIDERSCHEIT, 2005) pode-se entender a crescente popularização do Método Pilates, visto que o princípio fundamental do Método, segundo Muscolino e Cipriani (2004) é a centralização. A centralização explana que todo movimento não só começa, mas também é sustentado no centro do corpo humano, especificamente pelos músculos abdominais, paravertebrais lombares, flexores do quadril, extensores do quadril e assoalho pélvico, os quais formam uma estrutura que suporta e reforça o tronco, conhecida como core, e denominada, dentro do ambiente do Pilates, de power house (PILATES; MILLER, 1934; MUSCOLINO; CIPRIANI, 2004; ARAÚJO et al., 2010; MARÉS et al., 2012)

Tendo como foco trabalhar o power house, muitos exercícios do Método Pilates desafiam a estabilidade do centro através do movimento das regiões apendiculares. Dentre eles, pode-se citar o Leg Circles, um exercício amplamente utilizado nas aulas de Pilates. O exercício pode ser descrito como a circundução da articulação coxofemoral em círculos contínuos e fluídos, mantendo contraída a musculatura do power house (APARICIO e PEREZ, 2005; ISACOWITZ, 2006; SILER, 2008). Quando realizado no solo (ou mat, conforme denominado no método) os círculos são unilaterais, enquanto nos aparelhos, são bilaterais. Nos aparelhos Reformer, Cadillac e Wall Unit, tal exercício é realizado com molas, sendo que os dois últimos aparelhos permitem optar por diversas posições de fixação das molas. Sabe-se que, ao variar o ponto de fixação das molas, a realização de um mesmo exercício, pode ter diferentes respostas musculares, culminando em alterações na musculatura estabilizadora do tronco (LOSS et al., 2010), ou mesmo na inversão da relação agonistaantagonista (SILVA et al., 2009).

Sabendo da importância de uma correta ativação dos músculos estabilizadores do tronco, levanta-se o seguinte questionamento: Qual a altura de molas durante o exercício Leg Circles no Cadillac que mais ativa essa musculatura? Sendo assim, o presente estudo objetivou comparar a atividade elétrica de músculos do power house (reto abdominal, oblíquo externo, oblíquo interno/transverso abdominal e multífido) durante a execução do exercício Leg Circles no aparelho Cadillac com mola alta e com mola baixa.

\section{MÉTODOS}

Participaram do estudo 10 instrutoras de Pilates saudáveis (Tabela 1). A estimativa amostral foi calculada no software G*Power 3.1.9.2 a partir dos dados do estudo de Sacco et al. (2005), considerando um poder estatístico de $80 \%$ (power $(1-\beta$ err prob) $=0,95)$. Foram adotados os seguintes critérios de elegibilidade: ser instrutora de Pilates há, no mínimo, seis meses; estar praticando ao menos uma vez por semana no último ano; não apresentar dor lombar aguda ou crônica, dor nos membros inferiores ou na região abdominal; não ter assimetrias de membros inferiores ou tronco, identificadas visualmente. Essa pesquisa seguiu a resolução 466/2012 do Conselho Nacional de Saúde e foi aprovada pelo Comitê de Ética em Pesquisa da UFRGS (registrada na Plataforma Brasil sob o CAAE: 43223415.0.0000.5347).

Tabela 1. Caracterização da Amostra: Instrutoras de Pilates $(\mathrm{n}=10)$.

\begin{tabular}{cccc} 
& Média (desvio padrão) & Valor máximo & Valor mínimo \\
\hline Idade (anos) & $30( \pm 5)$ & 40 & 24 \\
Peso $(\mathrm{kg})$ & $58( \pm 7)$ & 68 & 49 \\
Estatura (cm) & $163( \pm 7)$ & 176 & 155 \\
Treino de Pilates (frequência semanal) & $2( \pm 1)$ & 4 & 1 \\
Tempo de prática ininterrupta (anos) & $5( \pm 5)$ & 17 & 1 \\
\hline
\end{tabular}


Inicialmente, as participantes assinaram o Termo de Consentimento Livre e Esclarecido, em seguida foram registrados peso, estatura, idade e informações sobre sua prática do Pilates. Foi solicitado a todas que realizassem o exercício como uma "familiarização" ao equipamento que seria utilizado (da marca Physio Pilates), para que relatassem a mola com resistência similar à que geralmente utilizam durante os seus treinos de Pilates e, caso necessário, se adequassem ao padrão respiratório solicitado. A partir de então foram colocados os eletrodos de superfície, em seguida, foram realizadas as Contrações Isométricas Voluntárias Máximas (CIVMs) de cada músculo, e por fim, dez repetições de círculos externos do exercício Leg Circles no Cadillac. A ordem de execução foi aleatória sistêmica - para as CIVMs e aleatória para o exercício. A execução foi supervisionada por uma instrutora de Pilates duplamente certificada no Método, experiente, graduada em Educação Física, especialista em Método Pilates, a qual orientou verbalmente que o power house fosse contraído ao longo das repetições.

O Leg Circle no Cadillac foi realizado em duas posições de mola, alta e baixa (Figura 1). A mola alta foi fixada barra horizontal superior do Cadillac e a mola baixa na barra móvel fixada na altura do tornozelo da participante quando em decúbito dorsal e aproximadamente 90 de flexão de quadril. O exercício iniciou com a participante em decúbito dorsal, com as alças de pés colocadas, sendo solicitado completo relaxamento do power house e dos membros inferiores. Após o posicionamento da participante, foi solicitada flexão de quadril até 90ำ, para que fossem iniciados os círculos externos bilaterais a partir da circundução da articulação coxo-femoral, na velocidade e amplitude normalmente utilizadas pela participante em seu treinamento, mantendo um padrão respiratório de inspirar na primeira metade do círculo (definida do pelo momento onde o quadril se encontra em maior amplitude de flexão até a menor amplitude de flexão) e expirar na outra metade (APARICIO; PEREZ, 2005; SILER, 2008). Foram realizadas dez repetições em cada posição de mola, com dois minutos de intervalo entre cada série.
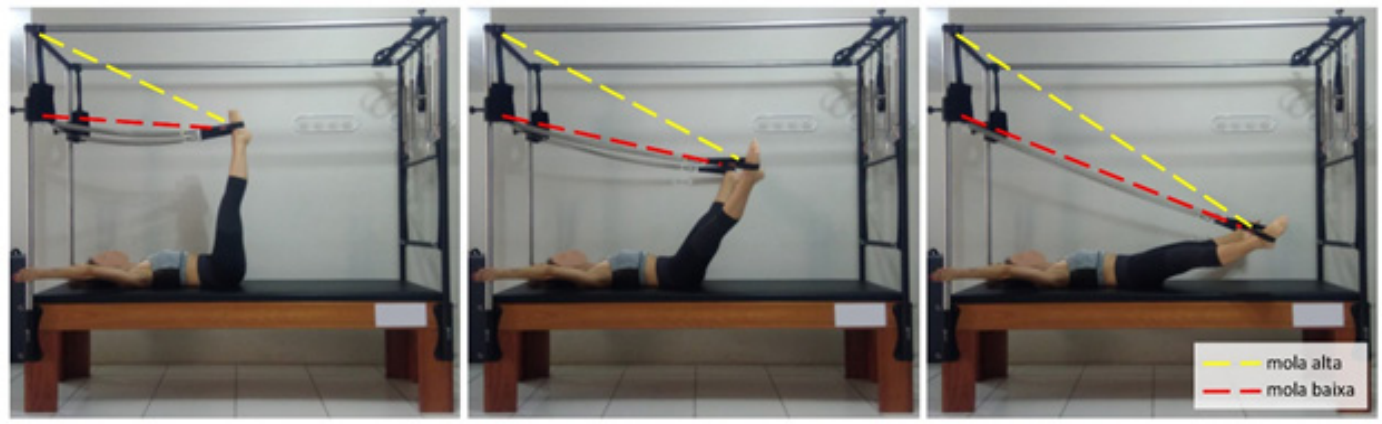

Figura 1. Fase concêntrica do Leg Circles no Cadillac representando duas posições de mola: alta e baixa.

A coleta dos dados foi realizada por uma Webcam com resolução de $1.280 \times 720$, taxa de amostragem de 30 $\mathrm{Hz}$ (Microsoft Lifecan HD-6000) sincronizada com a aquisição eletromiográfica. Os dados de eletromiografia foram coletados através de um sistema de aquisição de dados Miotool Wireless USB conectado ao Software Miotec Suite (Miotec Equipamentos Biomédicos Ltda, Porto Alegre, Brasil). O equipamento possui oito canais analógicos de entrada com taxa de amostragem de $2000 \mathrm{~Hz}$. Para aderência dos eletrodos e captação do sinal eletromiográfico foram observados rigorosamente todos os procedimentos recomendados pela Sociedade Internacional de Eletrofisiologia e Cinesiologia (ISEK) (MERLETTI, 1999), Sociedade Internacional de Biomecânica (ISB) (SODERBERG; KNUTSON, 2000) e projeto Surface ElectroMyoGraphy for the Non-Invasive Assessment of Muscles (SENIAM), (HERMENS et al., 2000).

Foram fixados os pares de eletrodos de superfície descartáveis (Kendall, Meditrace - 100; $\mathrm{Ag} / \mathrm{AgCl}, 10 \mathrm{~mm}$ de diâmetro, auto-adesiva, na configuração bipolar), sobre o ventre muscular, paralelo às fibras, separados $2 \mathrm{~cm}$ um do outro em locais previamente identificados. O par de eletrodos do multífido (MU) foi colocado alinhado com a espinha ilíaca póstero-superior no espaço intermediário entre a L1 e L2, ao nível do processo espinhal da vértebra $\mathrm{L} 5$ (2-3cm da linha média (HERMENS et al., 2000), os eletrodos do músculo oblíquo externo (OE) foram colocados conforme Escamilla et al.(2006), acima da espinha ilíaca ântero-superior no nível da cicatriz umbilical, os do oblíquo interno e transverso abdominal (OI/TrA) segundo Sniijders et al.(1998) a $2 \mathrm{~cm}$ da espinha ilíaca ântero-superior (dentro de um triângulo desenhado pelo ligamento inguinal, borda lateral do músculo reto abdominal e linha conectando as espinhas ilíacas ântero-superior), os eletrodos do reto abdominal (RA) foram colocados a $2 \mathrm{~cm}$ lateral da cicatriz umbilical, e o de referência na face anterior da clavícula (NEUMANN; GILL, 2002). Considerando simetria entre os lados, e a característica bilateral do exercício, todos os eletrodos foram colocados no lado direito. 
Para a normalização dos sinais, foram coletadas CIVMs de cinco segundos recebendo um incentivo verbal (KONRAD, 2006). Para a CIVM dos músculos abdominais, de acordo com o proposto por Konrad (2006), a participante foi posicionada em decúbito dorsal com uma flexão de tronco de aproximadamente $30^{\circ}$, mantendo o quadril e os joelhos flexionados a 90 e os pés apoiados sobre a maca. Para CIVM do RA foi solicitado que a participante fizesse flexão do tronco, para o OE, flexão do tronco com rotação para o lado esquerdo e para o Ol e transverso do abdome, flexão do tronco com rotação para o lado direito. Também segundo Konrad (2006) para a CIVM do MU, a participante foi posicionada em decúbito ventral, sendo solicitado que uma extensão do tronco. Todas as CIVMs foram realizadas duas vezes, com a voluntária amarrada, de forma a garantir a característica isométrica da contração. Foi respeitado o intervalo de dois minutos entre cada CIVM, o que se acreditou ser tempo suficiente para evitar a interferência da fadiga, uma vez que as participantes eram treinadas.

Os dados foram tratados no software BIOMECSAS (versão 1.0) do Grupo de Investigação da Mecânica do Movimento1. Para cada CIVM foi realizado um Envelope RMS com intervalo de 1 segundo e janelamento móvel do tipo Hamming. Para fins de normalização foi utilizado o maior valor de envelope de cada um dos músculos durante as CIVMs, sendo que os músculos flexores foram avaliados em todas as situações que envolviam flexão, foi assumido o maior valor de ativação encontrado. Para os exercícios, após a remoção do off set, o sinal foi filtrado utilizando um filtro digital Butterworth passa banda com frequências de corte entre $20 \mathrm{~Hz}$ e $500 \mathrm{~Hz}$, de quarta ordem. Cada curva foi recortada, dando origem a dez novas curvas, uma para cada repetição. O critério de recorte das curvas foi definido pelo vídeo, considerando o início de cada repetição o ponto de maior flexão do quadril. Visando evitar variações do efeito inercial, foram excluídas a primeira e a última execução de cada exercício. Para os exercícios foi utilizado o valor RMS de cada repetição. Assumiu-se como valor representativo da ativação muscular da participante a média das oito repetições de cada situação, o qual foi normalizado pelo valor normalizado pela CIVM do respectivo músculo.

A análise estatística foi realizada no software SPSS 20.0, o nível de significância adotado foi 5\%. A normalidade foi verificada pelo Teste de Shapiro-Wilk. Nos dados paramétricos (oblíquo externo) foi realizado o Teste T para dados pareados, em dados não paramétricos (reto abdominal, oblíquo interno e multífido), foi utilizado o Teste de Wilcoxon (FIELD, 2009). Todos os testes foram analisados de forma bicaudal, a exceção do multífido, visto que o estudo de Loss et al. (2010) fornece uma expectativa de que essa musculatura seja mais ativada na mola alta. O tamanho de efeito ( $r$ ) foi calculado segundo Field (2009), para ambos testes.

\section{RESULTADOS}

O diferente posicionamento das molas adotado no presente estudo afetou a atividade elétrica dos músculos OE, OI/TrA e MU. Entretanto, a diferença não se deu da mesma forma na medida em que o MU e o Ol/TrA foram mais ativados na mola alta, enquanto o OE na mola baixa (Figura 2). O OE foi mais ativado na mola baixa (Média 28,41; Erro Padrão 4,85) do que na mola alta (Média 5,11; Erro Padrão 0,75) apresentando grande tamanho de efeito T=-4,795, $p=0,001 ; r=0,84775$. O OI/TrA foi mais ativado na mola alta (Mediana 17,31) do que na mola baixa (Mediana 5,93) também com grande tamanho de efeito $z=-2,803 ; p<0,05 ; r=-0,62677$. Assim como o multífido que foi mais ativado na mola alta (Mediana 8,98) do que na mola baixa (Mediana 4,54) com tamanho de efeito razoável $z=-1,784 ; p<0,05, r=-0,39891$. Já o RA não apresentou diferença entre a mola alta (Mediana 2,44) e a mola baixa (Mediana 1,96) com baixo tamanho de efeito $z=-0,968 ; p>0,05, r=-0,21645$.

A maior atividade do MU durante o exercício realizado com a mola alta pode ser atribuída a um maior torque flexor da mola nesta situação. O torque flexor gerado pela mola baixa é menor do que o gerado pela mola alta, pois a mesma mola foi utilizada nas duas situações, e na fixação alta ela acaba sendo mais deformada. Ao ser mais deformada, tal situação gera mais torque flexor do que na fixação baixa. Melo e colaboradores (2011) avaliaram o torque de resistência da mola fixada em duas posições, durante a flexo-extensão do quadril no Cadillac, os resultados indicaram maior atuação dos MU na fixação mais alta avaliada, a qual corresponde à posição baixa do presente estudo. O que aparentemente justificaria que no presente estudo a cadeia muscular posterior, tanto da coxa quanto do tronco possa ser mais exigida na mola alta. Embora a musculatura extensora do quadril não tenha sido monitorada, esta mesma hipótese foi aventada por Loss e colaboradores (2010) que avaliaram os MU durante a flexo-extensão do quadril. Neste sentido, parece haver uma ação sinergista entre os músculos extensores do tronco e extensores do quadril - que são agonistas do movimento quando o torque da mola é maior do que o torque dos membros inferiores, o que, conforme apresentado, parece mais pertinente na fixação alta.

Ponderando a simetria do exercício, e analisando apenas os oblíquos de forma isolada, não há razões para acreditar que haja diferença entre os lados direito e esquerdo destes músculos. Desta forma, considerando-se que não há uma tendência de rotação do tronco para qualquer dos lados, as funções opostas dos oblíquos (GRAY; CARTER, 2011), rotação para o mesmo lado do OI e rotação para o lado oposto do OE, não parecem ser a justificativa para a diferença encontrada.

\footnotetext{
Disponível em: <http://www.ufrgs.br/biomec/>.
} 
No entanto há uma diferença nas inserções anatômicas de cada um destes músculos que poderia justificar os resultados. Ambos oblíquos se originam nas últimas costelas, mas enquanto o Ol se insere no púbis o OE se insere na crista ilíaca (GRAY; CARTER, 2011).

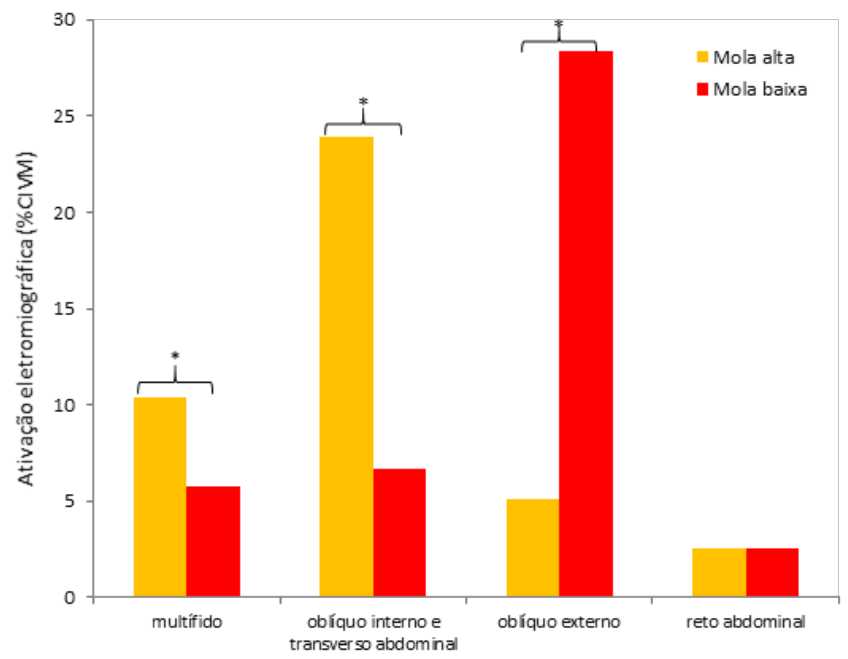

Figura 2. Ativação eletromiográfica durante execução do exercício Leg Circles realizado no Cadillac com mola alta e com mola baixa.

$* p<0,05$

A maior ativação do OE na mola baixa pode ser justificada pelas variações no torque gerado pelas diferentes fixações das molas, e pelas demais ações que estas variações desencadeariam. Além do OE, inserem-se na crista ilíaca importantes flexores de quadril como o reto femoral e o ilíaco (GRAY; CARTER, 2011), desta forma, parece razoável admitir uma ação sinérgica entre estes músculos. Dito de outra forma, mesmo que durante o exercício os participantes estejam voluntariamente tentando manter a pelve imóvel, diferentes tendências são provocadas, especialmente no que se refere ao movimento de retro e anteversão da pelve, gerada pela ativação dos flexores de quadril que nela se inserem. Conforme apresentado anteriormente, pode-se supor que em algumas fases do movimento, o torque gerado pela mola venha a ser menor do que o torque gerado pelo peso dos membros inferiores, o que exigiria uma atuação excêntrica de flexores do quadril. Como essa hipótese baseia-se em um menor torque flexor gerado pela mola, espera-se que tal situação seja encontrada na mola baixa, se não exclusivamente, ao menos de forma mais intensa (menor torque flexor da mola, e em mais amplitudes dos movimentos). Diante do exposto, supõe-se que com a mola baixa e consequentemente maior uso dos flexores de quadril, haveria uma tendência maior de anteversão, enquanto com a mola alta a tendência maior seria de retroversão, uma vez que conforme já explicado, pela maior deformação, a tendência flexora da mola seria maior do que na situação de mola baixa, exigindo mais uso de extensores do quadril.

Com relação ao oblíquo interno, é necessário salientar sua interação com o transverso do Abdome, uma vez que a ativação destes músculos não pode ser diferenciada no local onde foram posicionados os eletrodos (SNIIJDERS et al., 1998; MARSHAL; MURPHY, 2004). O transverso do abdome é um músculo profundo, e sua posição impossibilita uma medição isolada de sua ativação, com eletromiografia de superfície. No entanto, recentes estudos têm avaliado sua ativação juntamente com a ativação do oblíquo interno (LEE et al., 2013; BARBOSA et al., 2015; LEE; CHOI; LEE, 2015). Esses músculos, além de possuírem inervação comum, possuem também um tendão conjunto em sua inserção na crista púbica (GRAY; CARTER, 2011), o que leva a uma fusão de suas fibras no local onde se coloca os eletrodos de superfície para avaliar a ativação do Oblíquo interno (SNIIJDERS et al., 1998; MARSHAL; MURPHY, 2004). Ou seja, ao avaliar a ativação muscular na região inferomedial da espinha ilíaca ântero-superior, está se avaliando uma ativação conjunta do transverso do abdome e do obliquo interno (MARSHAL; MUPHY, 2003). Sabe-se que a avaliação eletromiográfica superficial do obliquo interno prediz a ativação do transverso do abdome, avaliada com eletrodos de inserção (McGILL; JUKER; KROPF, 1996) e que, mesmo ao avaliar tais músculos de forma individual, com ultrassom, eles agem em conjunto durante a execução de exercícios do Método, inclusive do leg circle (ENDLEMAN; DUNCAN, 2008). Ainda, segundo McGill, Juker e Kropf (1996) a ativação do Transverso do Abdome pode ser predita pela ativação do Oblíquo interno, aceitando um erro de 15\%.

Pode-se atribuir os resultados encontrados na mola alta para o OI/TrA, ao maior torque, e maior variação de torque 
gerado pela mola ao longo da execução. Maiores alterações no sentido e na magnitude do torque gerado pela mola provocariam uma maior instabilidade no tronco, sendo justificado, portanto, mais ativação dos estabilizadores profundos do tronco. A maior ativação do multífido nessa situação corrobora o exposto.

Com relação ao RA, sua ativação não variou, mantendo-se em aproximadamente $2,5 \%$ da CIVM em ambas situações. Pode-se supor que nenhuma das fixações de molas avaliadas gera demanda para tal músculo, o que pode ser associada a postura do tronco, que se mantém apoiado durante a execução do Leg Circles. Sendo o RA um flexor do tronco (GRAY; CARTER, 2011) e considerando que, em indivíduos saudáveis, espera-se uma ação estabilizadora dos músculos mais profundos, fica intuitivo prever a ativação encontrada com relação ao RA na população estudada.

O presente estudo corrobora os demais que avaliaram a musculatura do power house durante exercícios do Método (LOSS et al., 2010; SACCO;; MORI; QUEIROZ, 2014), demonstrando que não há uma ativação conjunta da musculatura do power house, como vem sendo preconizado (MUSCOLINO; CIPRIANI, 2004; RYDEARD; LEGER; SMITH, 2006). Apenas o comando verbal para "contrair o power house", adotada ao longo de todas as execuções talvez não seja suficiente para ativar a musculatura profunda da região quando a demanda mecânica é baixa. Uma vez que tal comando não foi o suficiente para ativar OI/TrA e MU na mola baixa, alguns exercícios do método são mais eficazes do que outros para ativar tais músculos e consequentemente desenvolver a estabilidade do tronco. É importante conhecer quais exercícios e quais variações são mais adequadas para trabalhar os estabilizadores, para que os tratamentos realizados com o Método Pilates possam ser ainda mais efetivos, podendo escolher cada exercício a partir dos objetivos de cada aluno. Além disso, a ativação correta desses músculos alivia a dor e diminui as recidivas da lombalgia (RICHARDSON et al., 2002, FRANÇA et al., 2008). Richardson et al. (2002) afirmam que se deve preconizar uma ativação do transverso do abdome isolado dos demais músculos flexores, como sendo a estratégia mais efetiva para o tratamento e prevenção da dor lombar

\section{CONSIDERAÇÕES FINAIS}

Considerando a importância da ativação dos músculos profundos do tronco, dentre as situações analisadas, a mola alta pode ser acatada como a posição mais adequada para ativar a musculatura profunda o tronco. A maior ativação o MU e do OI/TrA, somados à menor ativação do OE e a baixa ativação no RA encontrados em tal situação é o que se preconiza tanto em indivíduos saudáveis, quanto naqueles com algias ou com alguma patologia, pois a ativação profunda é a base do princípio da centralização.

No entanto, os resultados devem ser avaliados com cautela, uma vez que a amostra foi composta por indivíduos treinados. Mais estudos são necessários, no sentido de aprofundar o conhecimento desse exercício, e de conhecer os padrões de ativação muscular em outros exercícios, visto que a maior parte dos exercícios utilizados nas aulas de Pilates ainda não foi analisada.

\section{REFERÊNCIAS}

APARÍCIO, E.; PÉREZ, J. O autentico método pilates: a arte do controle. São Paulo: Planeta do Brasil, 2005.

ARAÚJO, M. E. A.; SILVA, E. B.; VIEIRA, P. C.; CADER, S. A.; MELLO, D. B.; DANTAS, E. H. M. Redução da dor crônica associada à escoliose não estrutural, em universitárias submetidas ao método Pilates. Motriz, Rio Claro, v. 16, n. 4, p. 958-66, 2010.

BARBOSA, A. W.; GUEDES, C. A.; BONIFÁCIO, D. N.; SILVA A. F.; MARTINS F. L.; BARBOSA M. C. A. The Pilates breathing technique increases the electromyographic amplitude level of the deep abdominal muscles in untrained people. Journal of Bodywork and Movement Therapies, London, v. 19, n. 1, p. 57-61, 2015.

ENDLEMAN, I.; CRITCHLEY, D. J. Transversus abdominis and obliquus internus activity during pilates exercises: measurement with ultrasound scanning. Archives of Physical Medicine and Rehabilitation, Reston, v. 89, n. 11, p. 2205-12, 2008.

ESCAMILLA R. F.; BABB E.; DEWITT R.; JEW P.; KELLEHER P.; BURNHAM T.; BUSCH J.; D’ANNA K, MOWBRAY R.; IMAMURA, R. T. Electromyographic analysis of traditional and nontraditional abdominal exercises: implications for rehabilitation and training. Physical Therapy, Alexandria, v. 86, n. 5, p. 656-71, 2006

FIELD, A. Descobrindo a estatística usando o SPSS-2. Porto Alegre: Artmed, 2009.

FRANÇA, F. J. R.; BURKE, T. N.; CLARET, D. C.; MARQUES, A. P. Estabilização segmentar da coluna 
lombar nas lombalgias: uma revisão bibliográfica e um programa de exercícios. Fisioterapia e Pesquisa, São Paulo, v. 15, n. 2, p. 200-6, 2008.

GRAY, H.; CARTER, H. V. Gray's Anatomy: descriptive and surgical. New York: Cosimo Classics, 2011.

HERMENS, H. J.; FRERIKS, B.; DISSELHORST-KLUG, C.; RAU, G. Development of recommendations for SEMG sensors and sensor placement procedures. Journal of Electromyography and Kinesiology, London, v. 10, n. 5, p. 361-74, 2000.

HIDES, J. A.; STOKES, M. J.; SAIDE, M. J. G. A.; JULL, G. A.; COOPER; D. H. Evidence of lumbar multifidus muscle wasting ipsilateral to symptoms in patients with acute/subacute low back pain. Spine, Philadelphia, v. 19, n. 2, p. 165-72, 1994.

HODGES, P. W.; RICHARDSON, C. A. Inefficient muscular stabilization of the lumbar spine associated with low back pain. A motor control evaluation of transversus abdominis. Spine, Philadelphia, v. 21, n. 22, p. 2640-50, 1996.

ISACOWITZ, R. Pilates. Champaign: Human Kinetics, 1nd ed 2006.

KONRAD, P. The ABC of EMG - a practical introduction to kinesiological electromyography. Scottsdale: Noraxon Inc, 2006.

LEE A. Y.; KIM E. H.; CHO Y. W.; KWON S. O.; SON S. M.; AHN S. H. Effects of abdominal hollowing during stair climbing on the activations of local trunk stabilizing muscles: a cross-sectional study. Annals of Rehabilitation Medicine, Seoul, v. 37, n. 6, p. 804-13, 2013.

LEE, T. H.; CHOI, J. D.; LEE, N. G. Activation timing patterns of the abdominal and leg muscles during the sit-to-stand movement in individuals with chronic hemiparetic stroke. Journal of Physical Therapy Science, Tokyo, v. 27, n. 11, p. 3593-5, 2015.

LOSS, J.; MELO, M. O.; ROSA, C. H.; SANTOS, H. B.; TORRE, M.; SILVA, Y. O. Electrical Activity of External Oblique and Multifidus Muscles During the Hip Flexion Extension Exercise Performed in the Cadillac With Different Adjustments of Springs and Individual Positions. Revista Brasileira de Fisioterapia, São Carlos, v. 14, n. 6, p. 510-7, 2010.

MARÉS, G.; OLIVEIRA, K. B. D.; PIAZZA, M. C.; PREIS, C.; BERTASSONI NETO, L. A importância da estabilização central no método Pilates: uma revisão sistemática. Fisioterapia em Movimento, Curitiba, v. 25, n. 2, p. 445-51, 2012

MARQUES, N. R.; MORCELLI, M. H.; HALLAL, C. Z.; GONÇALVES, M. EMG activity of trunk stabilizer muscles during Centering Principle of Pilates Method. Journal of Bodywork and Movement Therapies, London, v. 17, n. 2, p. 185-91, 2013.

MARSHALL, P. W.; MURPHY, Bernadette A. Core stability exercises on and off a Swiss ball. Archives of Physical Medicine and Rehabilitation, Reston, v. 86, n. 2, p. 242-9, 2004.

McGILL, S. M. Low back stability: from formal description to issues for performance and rehabilitation. Exercise and Sport Sciences Reviews, Philadelphia v. 29, n. 1, p. 26-31, 2001.

McGILL, S.; JUKER, D.; KROPF, P. Appropriately placed surface EMG electrodes reflect deep muscle activity (psoas, quadratus lumborum, abdominal wall) in the lumbar spine. Journal of Biomechanics, Ann Arbor, v. 29, n. 11, p. 1503-7, 1996.

MELO, O. M.; GOMES, L. E.; SILVA, Y. O.; BONEZI, A.; LOSS, J. F. Análise do torque de resistência e da força muscular resultante durante exercícios de extensão de quadril no Pilates e suas implicações na prescrição e progressão. Revista Brasileira de Fisioterapia, São Carlos, v. 15, n. 1, p. 23-30, 2011.

MERLETTI, R. Standards for reporting EMG data. Journal of Electromyography and Kinesiology, London, v. 9, n. 1, p. 3-4, 1999.

MUSCOLINO J. E.; CIPRIANI S. Pilates and "powerhouse"- I. Journal of Bodywork and Movement Therapies, London, v. 8, n. 1, p. 15-24, 2004.

NEUMANN, P.; GILL, V. Pelvic floor and abdominal muscle interaction: EMG activity and intraabdominal pressure. International Urogynecology Journal and Pelvic Floor Dysfunction, Burnsville, v. 13, n. 2 , p. $125-32,2002$.

PILATES, J. H.; MILLER, W.J. A Obra Completa de Joseph Pilates: Sua Saúde e Retorno a Vida Através da Contrologia. São Paulo: Phorte, 1934.

RICHARDSON C. A.; SNIJDERS C. J.; HIDES J. A.; DAMEN L.; PAS M. S.; STORM J. The relation between the transversus abdominis muscles, sacroiliac joint mechanics, and low back pain. Spine, Philadelphia, 
v. 27, n. 4, p. 399--405, 2002.

RICHARDSON, C. A.; JULL, G. A. Muscle control-pain control. What exercises would you prescribe? Manual therapy, Scotland, v. 1, n. 1, p. 2-10, 1995.

RYDEARD, R.; LEGER, A.; SMITH, D. Pilates-based therapeutic exercise: effect on subjects with nonspecific chronic low back pain and functional disability: a randomized controlled trial. Journal of Orthopaedic and Sports Physical Therapy, Alexandria, v. 36, n. 7, p. 472-84, 2006.

SACCO I. C. N.; MORI E. T. T.; QUEIROZ B. C.; MARCONI N.; PEREIRA I. L. R. Electromyographic assessment of trunk and shoulder muscles during a pilates pull-up exercise. Motriz, Rio Claro, v. 20, n. 2, p. 206-12, 2014.

SACCO, I. C. N.; ANDRADE, M. S.; SOUZA, P. S.; NISIYAMA, M.; CANTUÁRIA, A. L.; MAEDA, F. Y. I.; PIKEL, M. Método pilates em revista: aspectos biomecânicos de movimentos específicos para reestruturação postural - estudos de caso. Revista Brasileira de Ciência e Movimento, Brasília, v. 13, n. 4, p. 65-78, 2005.

SHERRY, M.; BEST, T.; HEIDERSCHEIT, B. The core: where are we and where are we going? Clinical Journal of Sport Medicine, Leawood, v. 15, n. 1, p. 1-2, 2005.

SILER, B. O corpo pilates. São Paulo: Summus, 2008.

SILVA Y. O.; MELO M. O.; GOMES L. E.; BONEZI A.; LOSS J. F. Análise da resistência externa e da atividade eletromiográfica do movimento de extensão de quadril realizado segundo o método Pilates. Brazilian Journal of Physical Therapy, Fortaleza, v. 13, n. 1, p. 82-8, 2009.

SNIJDERS C. J.; RIBBERS M. T.; BAKKER H. V.; STOECKART R.; STAM H. J. EMG recordings of abdominal and back muscles in various standing postures: validation of a biomechanical model on sacroiliac joint stability. Journal of Electromyography and Kinesiology, London, v. 8, n. 4, p. 205-14, 1998.

SODERBERG, G. L.; KNUTSON, L. M. A guide for use and interpretation of kinesiologic electromyographic data. Physical Therapy, Alexandria, v. 80, n. 5, p. 485-98, 2000.

Autor correspondente: Catiane Souza

E-mail: catiane-souza@hotmail.com 